\title{
Reduction in liver fat by dietary MUFA in type 2 diabetes is helped by enhanced hepatic fat oxidation
}

\author{
Lutgarda Bozzetto $^{1}$ - Giuseppina Costabile ${ }^{1}$ - Delia Luongo ${ }^{2}$ - Daniele Naviglio ${ }^{3}$. \\ Valentina Cicala ${ }^{1} \cdot$ Chiara Piantadosi $^{1} \cdot$ Lidia Patti $^{1} \cdot$ Paola Cipriano $^{1} \cdot$ \\ Giovanni Annuzzi ${ }^{1}$ - Angela A. Rivellese ${ }^{1}$
}

Received: 10 June 2016 / Accepted: 19 August 2016/Published online: 20 September 2016

(C) Springer-Verlag Berlin Heidelberg 2016

\begin{abstract}
Aims/hypothesis The aim of this work was to investigate hepatic lipid metabolic processes possibly involved in the reduction of liver fat content (LF) observed in patients with type 2 diabetes after an isoenergetic diet enriched in monounsaturated fatty acids (MUFAs).

Methods This is an ancillary analysis of a published study. In a parallel-group design, 30 men and eight women, aged 35-70 years, with type 2 diabetes and whose blood glucose was controlled satisfactorily $\left(\mathrm{HbA}_{1 \mathrm{c}}<7.5 \%[58 \mathrm{mmol} / \mathrm{mol}]\right)$ by diet or diet plus metformin, were randomised by MINIM software to follow either a high-carbohydrate/high-fibre/low-glycaemic index $\operatorname{diet}(\mathrm{CHO} /$ fibre diet, $n=20$ ) or a high-MUFA diet (MUFA diet, $n=18$ ) for 8 weeks. The assigned diets were known for the participants and blinded for people doing measurements. Before and after intervention, LF was measured by ${ }^{1} \mathrm{H}-\mathrm{MRS}$ (primary outcome) and indirect indices of de novo lipogenesis (DNL) (serum triacylglycerol palmitic:linoleic acid ratio), stearoyl-CoA desaturase activity (SCD-1) (serum triacylglycerol palmitoleic:palmitic acid ratio) and hepatic $\beta$-oxidation of fatty acids ( $\beta$-hydroxybutyrate plasma concentrations) were measured.
\end{abstract}

Electronic supplementary material The online version of this article (doi:10.1007/s00125-016-4110-5) contains peer-reviewed but unedited supplementary material, which is available to authorized users.

Giovanni Annuzzi

annuzzi@unina.it

1 Department of Clinical Medicine and Surgery, Federico II University, Via Pansini 5, 80131 Naples, Italy

2 Institute of Biostructures and Bioimaging, National Research Council, Naples, Italy

3 Department of Chemical Science, Federico II University, Naples, Italy
Results LF was reduced by $30 \%$ after the MUFA diet, as already reported. Postprandial $\beta$-hydroxybutyrate incremental AUC (iAUC) was significantly less suppressed after the MUFA diet $(n=16)(-2504 \pm 4488 \mu \mathrm{mol} / 1 \times 360 \mathrm{~min}$ vs baseline $-9021 \pm 6489 \mu \mathrm{mol} / 1 \times 360 \mathrm{~min})$ while it was unchanged after the $\mathrm{CHO} /$ fibre diet $(n=17)(-8168$ $\pm 9827 \mu \mathrm{mol} / \mathrm{l} \times 360 \mathrm{~min}$ vs baseline $-7206 \pm 10,005 \mu \mathrm{mol} /$ $1 \times 360 \mathrm{~min}, p=0.962)($ mean $\pm \mathrm{SD}, p=0.043)$. In the participants assigned to the MUFA diet, the change in postprandial $\beta$-hydroxybutyrate iAUC was inversely associated with the change in LF $(r=-0.642, p=0.010)$. DNL and SCD-1 indirect indices did not change significantly after either of the dietary interventions.

Conclusions/interpretation Postprandial hepatic oxidation of fatty acids is a metabolic process possibly involved in the reduction of LF by a MUFA-rich diet in patients with type 2 diabetes.

Trial registration ClinicalTrials.gov NCT01025856

Funding The study was funded by Ministero Istruzione Università e Ricerca and Italian Minister of Health.

Keywords De novo lipogenesis · Diet · Liver fat . Monounsaturated fatty acids $\cdot$ Stearoyl-coenzyme A desaturase-1 activity - Type 2 Diabetes $\cdot \beta$-Hydroxybutyrate . $\beta$-Oxidation

$\begin{array}{ll}\text { Abbreviations } \\ \text { DNL } & \text { De novo lipogenesis } \\ \text { iAUC } & \text { Incremental AUC } \\ \text { LF } & \text { Liver fat content } \\ \text { MUFA } & \text { Monounsaturated fatty acid } \\ \text { SCD1 } & \text { Stearoyl-coenzyme A desaturase-1 } \\ \text { SFA } & \text { Saturated fatty acid }\end{array}$




\section{Introduction}

Fatty liver is associated with type 2 diabetes [1] and dietary approaches useful for type 2 diabetes treatment also improve fatty liver [2]. We previously reported that an isoenergetic diet rich in monounsaturated fatty acids (MUFAs) induced a clinically relevant reduction $(-30 \%)$ in liver fat content (LF) in patients with type 2 diabetes [3]. The mechanisms behind this effect are unknown and may relate to the complex interactions between anabolic and catabolic hepatic pathways, including the following: (1) the uptake of NEFA derived from adipose tissue lipolysis, chylomicron remnants and intravascular hydrolysis of triacylglycerol-rich lipoproteins; (2) post-absorptive de novo lipogenesis (DNL), which is $15-25 \%$ higher in people with fatty liver and stimulated under conditions of excess carbohydrate intake [4]; (3) desaturation of saturated fatty acids (SFAs) to MUFAs by stearoyl-CoA desaturase (SCD-1), a process that is linked to DNL and occurs to a greater extent in individuals with fatty liver, likely being an adaptation to the excessive availability of SFAs [5]; (4) $\beta$-oxidation of fatty acids and ketogenesis, which are increased in people with obesity and hypertriglyceridaemia and likely prevent excess fat deposition in the liver [5] and (5) fatty acid secretion as circulating VLDL-triacylglycerols.

In this ancillary analysis of a published randomised controlled trial [3], we evaluated hepatic DNL, SCD1 activity and $\beta$-oxidation in a cohort with type 2 diabetes in whom a MUFA-rich diet resulted in a significant reduction of LF.

\section{Methods}

Participants Individuals with type 2 diabetes of both sexes were recruited at Federico II University Medical School Hospital and enrolled after giving their written informed consent. Participants were overweight or obese, without body weight changes over the previous 6 months, had satisfactory blood glucose control $\left(\mathrm{HbA}_{1 \mathrm{c}}<7.5 \%[58 \mathrm{mmol} / \mathrm{mol}]\right)$ by means of diet or metformin plus diet, were not on lipid-lowering drugs and had no evidence of liver disease apart from non-alcoholic steatosis. The main characteristics of the participants in this study $(n=33)$ are reported in ESM Table 1.

The trial design was approved by the Federico II University Ethics Committee and registered at ClinicalTrials.gov (registration no. NCT01025856).

Study design In a $2 \times 2$ parallel-group factorial design, after a 3 week run-in on a diet similar to their habitual diet (carbohydrate $48 \%$, total fat $33 \%$, saturated fat $13 \%$ and protein $18 \%$ of total energy intake), participants were randomly assigned to one of the following 8 week interventions: (1) high-carbohydrate/high-fibre/low-glycaemic index diet (CHO/ fibre diet); (2) high-MUFA diet (MUFA diet); (3) CHO/fibre diet plus physical training; (4) MUFA diet plus physical training [3]. The assigned experimental diets were isoenergetic in order to keep body weight constant and differed in macronutrient composition for $\mathrm{CHO} /$ fibre diet vs MUFA diet, respectively: carbohydrate $52 \%$ vs $40 \%$, fibre 28 vs $10 \mathrm{~g} /$ $4184 \mathrm{~kJ}$, glycaemic index (bread reference) $60 \%$ vs $95 \%$, fat $30 \%$ vs $42 \%$ and MUFA $16 \%$ vs $28 \%$. The diets followed are reported in ESM Table 2. Since in the original trial, light physical training was found to have no significant effect on LF, in the present study data were pooled according to diet only (i.e. $\mathrm{CHO} /$ fibre diet or MUFA diet), independently of the physical training, which was performed by ten participants in the $\mathrm{CHO} /$ /fibre-diet group and eight participants in the MUFA diet group [3]. Before and after intervention, anthropometrics, LF measurement (proton magnetic resonance spectroscopy, ${ }^{1} \mathrm{H}-\mathrm{MRS}$ ) and blood sampling before and after a test meal were performed. Test meals had a similar composition to the habitual diet of the study population at baseline, but were high in $\mathrm{CHO} /$ fibre or MUFA, according to the assigned experimental diet, after the intervention [3].

$\beta$-oxidation of fatty acids was assessed by measuring plasma $\beta$-hydroxybutyrate concentrations before and 180 and $360 \mathrm{~min}$ after the test meals [6]. DNL was assessed by calculating the ratio of palmitoleic to palmitic acid (C16:1/ C16:0) in fasting serum triacylglycerols [4]. SCD-1 activity was assessed by calculating the ratio of palmitic to linoleic acid (C16:0/C18:2n6) in fasting serum triacylglycerols [7].

Laboratory methods Plasma $\beta$-hydroxybutyrate concentrations were evaluated by an enzymatic endpoint method (DiaSys Diagnostic Systems, Holzheim, Germany) on an automated photometric analyser (ABX-Pentra 400; Horiba Medical, Kyoto, Japan). Samples were analysed in triplicate; normal and pathological reference samples were assayed in each batch for internal quality control. Palmitic, linoleic and palmitoleic acid levels in serum triacylglycerol fraction were analysed by gas chromatography. Briefly, serum lipids were extracted and triacylglycerols isolated by solid-phase extraction by standard procedures [8]. Methyl esters of fatty acids of triacylglycerols were prepared and analysed by gas chromatography with flame ionisation (DANI Instruments GC 1000, Cologno Monzese, Italy).

Statistical analysis Data are expressed as means \pm SD unless otherwise stated. Postprandial $\beta$-hydroxybutyrate incremental area under the curve (iAUC) was calculated by the trapezoidal rule as the $0-6 \mathrm{~h}$ AUC minus the area below the fasting value [9].

Between-treatments differences were evaluated by ANCOVA general linear model taking the 8 week values as dependent variables, the diet treatments as fixed factor and 
baseline values as covariates. Within groups, before-after intervention differences were evaluated by paired $t$ test. Bivariate associations were assessed by Pearson correlation. A $p$ value $<0.05$ was considered significant. Statistical analysis was performed according to standard methods using the SPSS software 20.0 (SPSS/PC; IBM, Armonk, NY, USA).

\section{Results}

At baseline, the MUFA or CHO/fibre groups did not differ significantly for age, BMI, fasting plasma glucose and lipids concentrations or $\mathrm{HbA}_{1 \mathrm{c}}$ (ESM Table 1). As previously reported [3], LF was decreased by a significantly greater extent after the MUFA diet than after the $\mathrm{CHO} /$ fibre diet $(-27 \pm 30 \%$ vs $-6 \pm 25 \%$, respectively, $p=0.034)$. Moreover, $\mathrm{HbA}_{1 \mathrm{c}}$ and fasting plasma triacylglycerol concentrations improved after the MUFA diet [3].

DNL The DNL index, measured as the ratio of palmitic to linoleic acid concentration, did not change after either the MUFA diet ( $4.18 \pm 2.20$ vs baseline $4.39 \pm 2.59, p=0.799)$ or the $\mathrm{CHO} /$ fibre $\operatorname{diet}(3.35 \pm 1.78$ vs baseline $4.23 \pm 2.69$, $p=0.176$ ).

Desaturase activity SCD-1 activity, measured as the ratio of palmitoleic to palmitic acid concentration, did not change after either the MUFA diet $(0.17 \pm 0.16$ vs baseline $0.19 \pm 0.20$, $p=0.978)$ or the $\mathrm{CHO} /$ fibre diet $(0.17 \pm 0.13$ vs baseline $0.23 \pm 0.29, p=0.424)$.

Fatty acid $\beta$-oxidation Figure 1 presents the fatty acid $\beta$-oxidation outcomes. Fasting plasma concentrations of $\beta$-hydroxybutyrate decreased significantly after the MUFA diet $(65 \pm 16$ vs baseline $84 \pm 24 \mu \mathrm{mol} / 1, p=0.015)$ but did not change after the $\mathrm{CHO} /$ fibre diet $(80 \pm 45$ vs baseline 78 $\pm 32 \mu \mathrm{mol} / 1, p=0.860$ ), with a non-significant difference between the two diets $(p=0.083)$. $\beta$-oxidation was suppressed during the postprandial period both at baseline and after dietary treatments, as shown by the lower concentrations of $\beta$-hydroxybutyrate after meals. Postprandial $\beta$-hydroxybutyrate iAUC was significantly less suppressed after the MUFA diet $(-2504 \pm 4488$ vs baseline $-9021 \pm 6489 \mu \mathrm{mol} / 1 \times 360 \mathrm{~min}$, $p=0.003$ ), while it remained unchanged after the $\mathrm{CHO} /$ fibre diet $(-8168 \pm 9827$ vs baseline $-7206 \pm 10,005 \mu \mathrm{mol} / 1 \times 360 \mathrm{~min}$, $p=0.962$ ), with a significant difference between the two diets $(p=0.043)$.

In the MUFA diet group, the change in postprandial $\beta$-hydroxybutyrate iAUC induced by the intervention was inversely associated with the change in LF $(r=-0.642$, $p=0.010$ ) as shown in Fig. 2.

\section{Discussion}

In this study, postprandial hepatic fat oxidation was enhanced by an 8 week MUFA-rich diet resulting in significantly reduced $\mathrm{LF}$, compared with a $\mathrm{CHO} /$ fibre diet. The postprandial suppression of $\beta$-oxidation was associated with a greater reduction in LF induced by the MUFA diet.

As a marker of hepatic $\beta$-oxidation we used plasma $\beta$-hydroxybutyrate concentration. NEFA oxidation generates intramitochondrial acetyl-CoA, which may either enter the Krebs cycle for oxidation to $\mathrm{CO}_{2}$ or produce $\beta$-hydroxybutyrate. The proportional contribution of ketogenesis to the overall NEFA oxidation is still unclear, with a higher partitioning to ketogenic pathways in obese individuals than in lean ones [10]. Kinetic studies indicate that a postprandial increase in
Fig. 1 (a, b) $\beta$-hydroxybutyrate $(\beta-\mathrm{OHB})$ plasma concentrations before and after the test meal at baseline (dashed line) and end of intervention (solid line) in the $\mathrm{CHO}$ /fibre group (a) and MUFA group (b). (c, d) Postprandial $\beta$-OHB iAUC at baseline and end of intervention in the $\mathrm{CHO} /$ fibre group (c) and MUFA group (d). Data are shown as means \pm SE. $* p<0.05$ vs baseline (paired sample $t$ test). ${ }^{\S} p=0.043$ for differences between changes in the two groups by ANCOVA
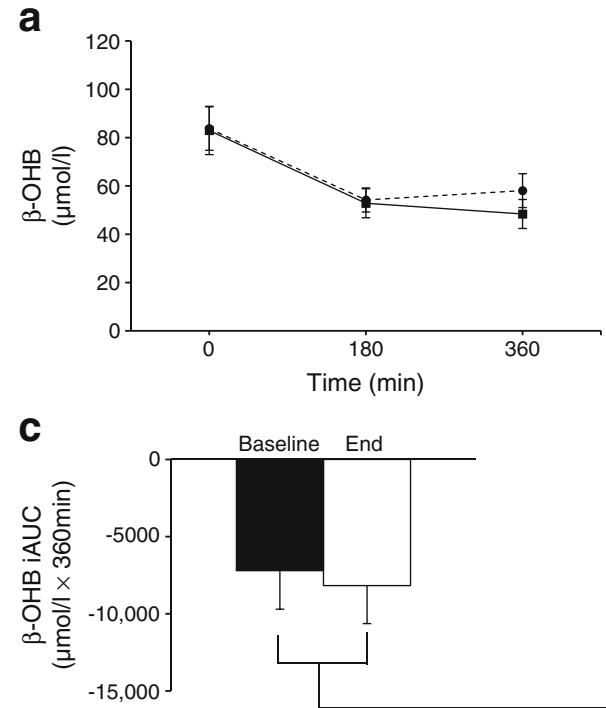

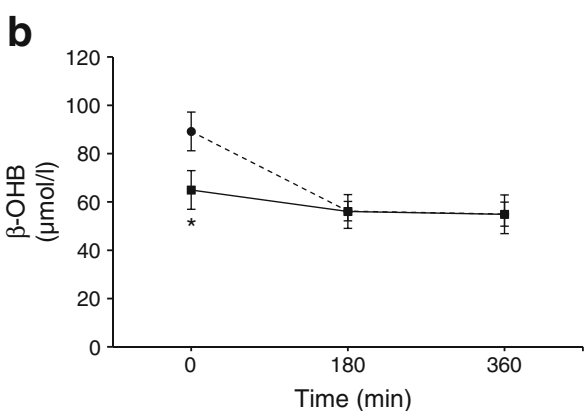

d

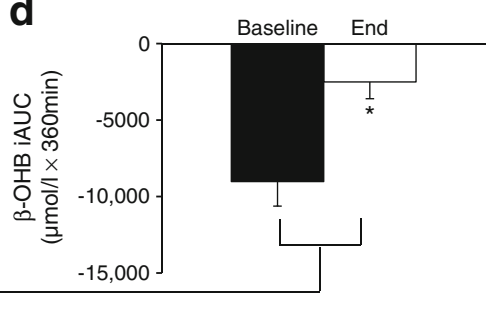


Fig. 2 Relationship between absolute changes $(\Delta, 8$ weeks minus baseline) in postprandial $\beta$-hydroxybutyrate iAUC and LF after the $\mathrm{CHO} /$ fibre diet $(n=16$, $r=-0.27, p=0.930)$ (a) and the MUFA diet $(n=15, r=-0.642$, $p=0.010)(\mathbf{b})$
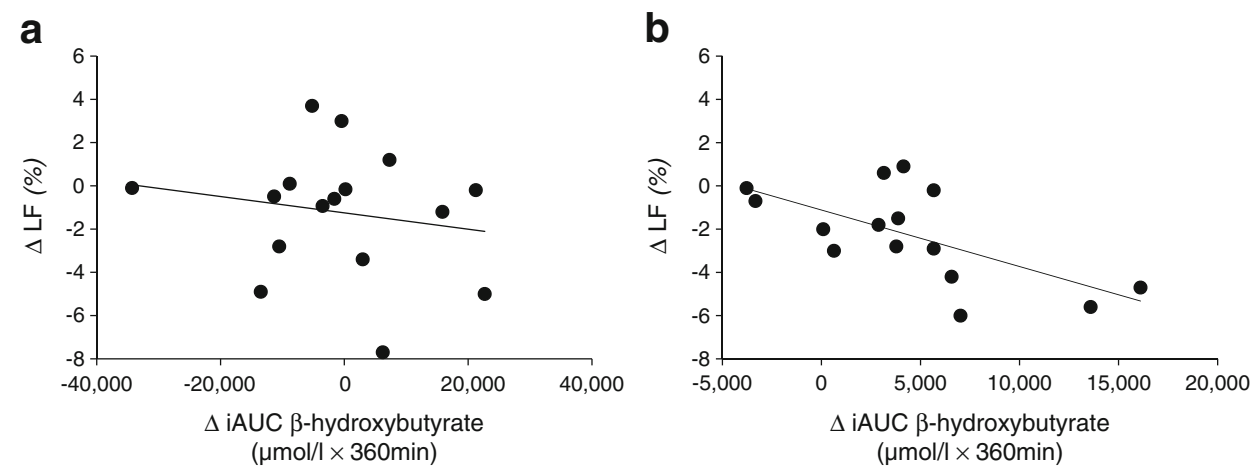

$\beta$-hydroxybutyrate production may be an adaptive mechanism to prevent $\mathrm{LF}$ accumulation in individuals needing to handle an energy oversupply. In line with this, in our study the increase in $\beta$-oxidation during the postprandial phase directly correlated with LF reduction in the MUFA diet group, corroborating the hypothesis that increasing fat oxidation is a main pathway to avoidance or reduction of liver fat deposition.

The increase in postprandial $\beta$-oxidation well integrates with the pleiotropic metabolic effects already shown for dietary MUFA. In the intestine, MUFAs are preferentially incorporated into a greater number of larger chylomicrons, which are hydrolysed faster than the smaller ones. This produces a higher proportion of MUFA in the postprandial NEFA pool more prone to oxidation, and therefore less prone to deposition, with respect to other types of fat [11]. Another likely mechanism underlying the effects of MUFAs on LF is that dietary MUFAs reaching the liver may be preferentially incorporated in VLDL and exported to adipose tissue [11]. Consistent with these observations, in our study the MUFA diet increased postprandial triacylglycerol concentrations in the chylomicron plus VLDL fraction, as previously reported [12].

No significant effects of the dietary intervention were observed on the indices of DNL and SCD-1 activity, indicating that these processes may not be directly involved in the reduction of LF by dietary MUFA. The lack of effect on the DNL and desaturase pathways, which are related to increased LF after very-high-carbohydrate intake [4], suggests that the reduction in LF observed in our study was unlikely to be related to the slightly lower carbohydrate content of the MUFA diet than the $\mathrm{CHO} /$ fibre diet [2].

A limitation of this study is the use of indirect markers of DNL and SCD-1 activity. Moreover, although $\beta$-hydroxybutyrate is widely accepted as a reliable marker of $\beta$-oxidation, its unstable chemical nature and the fact that its plasma concentrations result from the net balance between hepatic production and peripheral utilisation may represent some limitations for the interpretation of our results.

In conclusion, the reduction in LF obtained in individuals with type 2 diabetes receiving a MUFA-rich diet is associated with the modulation of the postprandial metabolic environment towards usage, rather than storage, of energy oversupply through the stimulation of postprandial hepatic oxidation of fatty acids.

Acknowledgements LB is grateful to the Italian Diabetes Society for the research grant 'Borsa di studio annuale SID-AMD Pasquale di Coste'.

Funding This study was funded by grants from 'Ministero Istruzione Università e Ricerca' (PRIN 2010-2011 -2010JCWWKM) and the Italian Ministry of Health (RF2010-2314264).

Duality of interest The authors declare that there is no duality of interest associated with this manuscript.

Contribution statement LB and GC designed the experiment, carried out the study, collected and analysed data and wrote the manuscript. GA designed the experiment and wrote the manuscript. DL, DN, VC and LP performed biochemical measurements, analysed the data and revised parts of the manuscript. CP and PC acquired data and revised parts of the manuscript. AAR designed the experiment and revised the manuscript. AAR is the guarantor of this work and, as such, had full access to all the data in the study and takes responsibility for the integrity of the data and the accuracy of the data analysis. All authors read and approved the final manuscript.

\section{References}

1. Bozzetto L, Prinster A, Mancini M et al (2011) Liver fat in obesity: role of type 2 diabetes mellitus and adipose tissue distribution. Eur $\mathrm{J}$ Clin Invest 41:39-44

2. Yki-Järvinen H (2015) Nutritional modulation of non-alcoholic fatty liver disease and insulin resistance. Nutrients 7:9127-9138

3. Bozzetto L, Prinster A, Annuzzi G et al (2012) Liver fat is reduced by an isoenergetic MUFA diet in a controlled randomized study in type 2 diabetic patients. Diabetes Care 35:1429-1435

4. Chong MF, Hodson L, Bickerton AS et al (2008) Parallel activation of de novo lipogenesis and stearoyl-CoA desaturase activity after 3 d of high-carbohydrate feeding. Am J Clin Nutr 87:817-823

5. Hodson L, Frayn KN (2011) Hepatic fatty acid partitioning. Curr Opin Lipidol 22:216-224 
6. Kotronen A, Seppälä-Lindroos A, Vehkavaara S et al (2009) Liver fat and lipid oxidation in humans. Liver Int 29:1439-1446

7. Peter A, Cegan A, Wagner S et al (2009) Hepatic lipid composition and stearoyl-coenzyme A desaturase 1 mRNA expression can be estimated from plasma VLDL fatty acid ratios. Clin Chem 55: 2113-2120

8. Burdge GC, Wright P, Jones AE, Wootton SA (2000) A method for separation of phosphatidylcholine, triacylglycerol, non-esterified fatty acids and cholesterol esters from plasma by solid-phase extraction. Br J Nutr 84:781-787

9. Burden RL, Faires JD (2011) Numerical analysis, 9th edn. Brooks/ Cole Boston, MA USA
10. Hodson L, McQuaid SE, Humphreys S et al (2010) Greater dietary fat oxidation in obese compared with lean men: an adaptive mechanism to prevent liver fat accumulation? Am J Physiol Endocrinol Metab 299:E584-E592

11. Bergouignan A, Momken I, Schoeller DA, Simon C, Blanc S (2009) Metabolic fate of saturated and monounsaturated dietary fats: the Mediterranean diet revisited from epidemiological evidence to cellular mechanisms. Prog Lipid Res 48:128-147

12. Bozzetto L, Annuzzi G, Costabile G et al (2014) A CHO/fibre diet reduces and a MUFA diet increases postprandial lipaemia in type 2 diabetes: no supplementary effects of low-volume physical training. Acta Diabetol 51:385-393 